\title{
Digital stacking of Tech Pan films and the photometry of faint galaxies
}

\author{
J.-M. Schwartzenberg ${ }^{1}$, S. Phillipps ${ }^{1}$ and Q. Parker ${ }^{2}$ \\ 1 Astrophysics Group, Department of Physics, University of Bristol, Bristol BS8 1TL, UK \\ jms@astro.cf.ac.uk, sxp@siva.bris.ac.uk \\ 2 UKST, Anglo-Australian Observatory, Siding Spring Mountain, Coonabarabran, NSW 2357, Australia \\ qap@aaocbn3.aao.gov.au
}

Received August 17; accepted October 3, 1995

\begin{abstract}
We describe the processing and co-addition of digitised scans of Tech Pan films, and discuss the scientific and photometric gains obtained from use of co-added films. This work is based on SuperCOSMOS scans at $10 \mu \mathrm{m}$ resolution of small regions of the Virgo cluster, which form part of a large-scale survey for low surface brightness galaxies in Virgo. Use of co-added Tech Pan films allows the detection and surface photometry of such galaxies to very faint limits, and therefore provides an invaluable tool for a deeper sampling of the cluster galaxy luminosity function over large areas of sky. The calibration steps necessary before the films can be stacked are described in detail, as well as the stacking process itself. Median-stacking is found to be more effective than averaging or simple adding of individual images, mainly due to its ability in removing spurious features present on single films. We also discuss several of the properties of stacked Tech Pan films, such as background variations, the extent of galaxy profiles and the detectability of small, faint galaxies.
\end{abstract}

Key words: image processing — galaxies: photometry — galaxies — clusters: Virgo cluster

\section{Introduction}

Since early 1993 the UK Schmidt Telescope (UKST) has used high resolution, high quantum efficiency Kodak Tech Pan film on a regular basis, in parallel with continued use of standard IIIa emulsion on glass (e.g. Parker et al. 1994). The advantages of Tech Pan over conventional emulsions are numerous and have been discussed in detail by Phillipps \& Parker (1993, hereafter PP93). They include an improved detective quantum efficiency (a gain of a factor 3-4 in DQE compared with standard Kodak III-a emulsions), extremely fine grain yielding improved resolution and remarkable uniformity. These advantages translate into a direct photometric gain of order 1 magnitude relative to equivalent sky-limited exposures on IIIa plates.

In recent years, co-addition of digitised photographic plates has proved useful in revealing faint galaxy features (Kemp \& Meaburn 1993). Experiments by Hawkins (1991) and Bland-Hawthorn et al. (1993) have shown that the gain in signal-to-noise follows approximately Poissonian statistics, with the noise on co-added frames decreasing

Send offprint requests to: J.M. Schwartzenberg as the square-root of the total exposure time. Plates have also been co-added photographically (e.g. Impey et al. 1988), offering gains in depth and contrast but without the advantage of digitisation, which permits a more quantitative, controlled and automated treatment of the data.

In this paper we demonstrate for the first time the feasibility and advantages of using stacked digitised scans of Tech Pan films from the UKST for the purpose of faint galaxy photometry. This work is preliminary to a new, large survey for low surface brightness galaxies in the Virgo cluster which will use co-added data from multiple Tech Pan exposures of the cluster. The aims of the survey, which will sample smaller and lower surface brightness galaxies than those in existing catalogues, have been set out in Schwartzenberg et al. (1995a, hereafter SPP95). Here we discuss the various reduction steps involved in the data processing, as well as the co-addition itself and its outcome in terms of higher signal-to-noise ratios, improved limiting isophotes and so forth.

Section 2 of this paper presents the observations and decribes the processing steps involved, and Sect. 3 is a comparison of the photometric properties of single and 
co-added Tech Pan films. Our conclusions are summarised in Sect. 4.

\section{Observations and data processing}

PP93 have discussed in detail the photometry of faint galaxies in the Virgo cluster using individual Tech Pan films taken with the $1.2 \mathrm{~m}$ UKST, small areas of which had been scanned using the now de-commissioned COSMOS automatic plate scanner at the Royal Observatory, Edinburgh (MacGillivray \& Stobie 1984). The work described here is based on new SuperCOSMOS scans of six separate film exposures centred at $12^{\mathrm{h}} 34^{\mathrm{m}},+11^{\circ} 12^{\prime}$ and covering the same $6^{\circ} \times 6^{\circ}$ region in the south-east of the Virgo cluster. Exposure times generally range from 60 to 75 min, with one film (used in PP93) having had an exceptionally long exposure (100 $\mathrm{min})$. All observations were made through a standard OG590 R-band filter. A log of the observations is shown in Table 1.

Table 1. Log of the UKST Virgo cluster Tech Pan observations

\begin{tabular}{cccc}
\hline Exposure No. & Date & $\begin{array}{c}\text { Exp. time } \\
(\text { min })\end{array}$ & $\begin{array}{c}\text { Seeing } \\
\text { FWHM }\left(^{\prime \prime}\right)\end{array}$ \\
\hline OR14310 & 16 Apr 1991 & 75 & 2.3 \\
OR14322 & 20 Apr 1991 & 100 & 2.5 \\
OR14782 & 28 Feb 1992 & 60 & 3.1 \\
OR14805 & 09 Mar 1992 & 60 & 2.9 \\
OR14811 & 10 Mar 1992 & 60 & 2.7 \\
OR14820 & 12 Mar 1992 & 70 & 2.7 \\
\hline
\end{tabular}

\subsection{Digitisation and its effects}

Digitisation clearly offers considerable advantages when using photographic material for faint object searches, as it allows direct numerical addition, manipulation and processing of individual exposures without the need to rely on photographic coaddition or amplification techniques. Use of a digitised image also enables the automated detection of large numbers of small-scale objects which would otherwise not be detected by eye, while analysis of digitised image parameters is the only possible way of obtaining accurate photometric profiles for objects of interest. By digitising several plate or film exposures we can therefore benefit from some of the advantages of linear detectors in terms of depth of imaging, while retaining the large area coverage afforded by photographic detectors on Schmidt telescopes (e.g. Kemp \& Meaburn 1993). Bland-Hawthorn et al. (1993) have recently emphasised the advantages of photographic digital stacking over CCD mosaics in terms of telescope time. This is particularly true with the greater depth and resolution of Tech Pan films.
The films listed in Table 1 have been digitised using the SuperCOSMOS machine (the new upgraded replacement for COSMOS, see Miller et al. 1992). The spot size and pixel size were both $10 \mu \mathrm{m}$, corresponding to a scale of 0.67 arcsec at the telescope. Only the central $5^{\circ} \times 5^{\circ}$ area of each film was scanned, as a strong vignetting pattern is present in the outer $1^{\circ}$ strips.

As a complete scan of 25 square degrees generates a total of $(26810)^{2}$ pixels, or $1.5 \mathrm{~Gb}$ of data, all processing and analysis have been performed on small subsets, which we have chosen to be of size $(2048)^{2}$ pixels. For the present work we have used five such subsets, each centred on one of the calibrating galaxies of PP93.

Due to the SuperCOSMOS scanning process - which measures the intensity of objects by recording the fraction of light from the scanning spot transmitted by each image - the background typically has a large number of counts and bright objects have low counts. For small variations about the sky level the density is closely linear with intensity (cf. Cawson et al. 1987). At the bright end this linearity extends to at least $21 R$ mag $\operatorname{arcsec}^{-2}(\mathrm{R} \mu)$, so rather than carry out a full density to intensity calibration via the characteristic curve (as in PP93) we have simply mirrored all values about the sky background.

\subsection{Sky subtraction}

One of the most important steps of the data reduction procedure is the sky subtraction - it is crucial to have as flat a background as possible if we are to detect objects with central intensities of only a few percent of sky (e.g. Irwin et al. 1990; Davies et al. 1994; Schwartzenberg et al. 1995b). Furthermore, the sky background needs to be relatively flat for the median-stacking process to work efficiently. Background variations across a frame may be caused by several factors, such as the presence of a bright star on or near the frame or irregularities in the emulsion. Fortunately Tech Pan emulsions appear considerably more uniform than conventional photographic plates (Parker et al. 1994), though some irregularities may still be introduced at the hypering/drying stage. In addition, the high DQE of Tech Pan films means that the outer regions of the point spread function of bright stars are much more extended and noticeable. Confusion of these outer regions with low surface brightness galaxy features can be averted at the detection stage by deleting the regions of a frame occuied by bright stars and their halos. These regions occupy typically less than $2 \%$ of each frame.

Extensive analysis of the effects of median filtering on other digital data (Schwartzenberg et al. 1995b; Driver et al. 1994) has shown that subtraction of a spatially medianfiltered background map considerably reduces the level of background variations. For each frame, we therefore created a map in which each pixel had the median value of the pixels in the original frame in an $n \times n$ box surrounding the pixel in question. This map was then subtracted 
from the original frame, producing a sky-subtracted image. After extensive experimentation a median filter box size of $256 \times 256$ pixels was chosen, as it provided the best representation of the existing background variations.

We must remember, however, that the median filtering procedure has the disadvantage of forcing an upper limit to the size of objects which can be detected: faint objects of scale size $\approx$ filter box size are lost, or at least severely degraded, in the process. At the distance of Virgo, however, 256 pixels (170 arcsec) correspond to at least $12 \mathrm{~h}^{-1}$ kpc (Jacoby et al. 1992), and therefore we may assume that median-filtering will not hamper the detection of the numerous, small-scale low surface brightness galaxies of interest (SPP95). Potentially interesting, larger galaxies lost in the filtering process will be retrieved at a later stage by inspection of the background maps created.

\subsection{Scaling}

For reasons discusssed in Sect. 2.5, we have decided to "co-add" the data via median-stacking rather than pure addition as adopted in previous photographic studies (e.g. Hawkins 1991, Kemp \& Meaburn 1993). Before stacking, therefore, it is necessary to further process all scans so as to bring them to a common scale. Several factors, both observational (changes in sky brightness, cloud cover, atmospheric seeing) and photographic (storage time, hypering of the emulsion, chemical fog level and scanning conditions) can cause each film scan to have different measured sky counts. Furthermore, differences in the shape of the characteristic curve (as a result of slight hypering and/or processing variations) and in the scanning set-up will likely alter the scaling between the grey levels and true intensity. Prior to stacking we have therefore applied both a multiplicative and an additive scaling factor to each film. The reference film chosen for this scaling was OR14310, which appeared to have the best signal to noise ratio and was also used in PP93.

In order to calculate the multiplicative scaling factors for the other five films, we matched the radial intensity profiles of IC3430 (cf. PP93) derived from every other film to that obtained from the reference frame, that is we scaled them so that the surface brightness from each film corresponded to the same number of counts above sky. The normalization point was chosen at $20^{\prime \prime}$ from the centre of the galaxy, in the linear region of the profile, i.e. that unaffected by any nuclear component. This should remove any possible problem due to different atmospheric seeing on the various films. The six individual profiles of IC3430 are shown in Fig. 1.

Profiles of the other calibrating galaxies from PP93 were also obtained from the median-stacked frames, to test the reliability of our scaling factors. These have turned out to be accurate for all the galaxies profiled, including VCC1017 and VCC1336 which have irregular profiles (see PP93). The fact that these five galaxies are spaced well

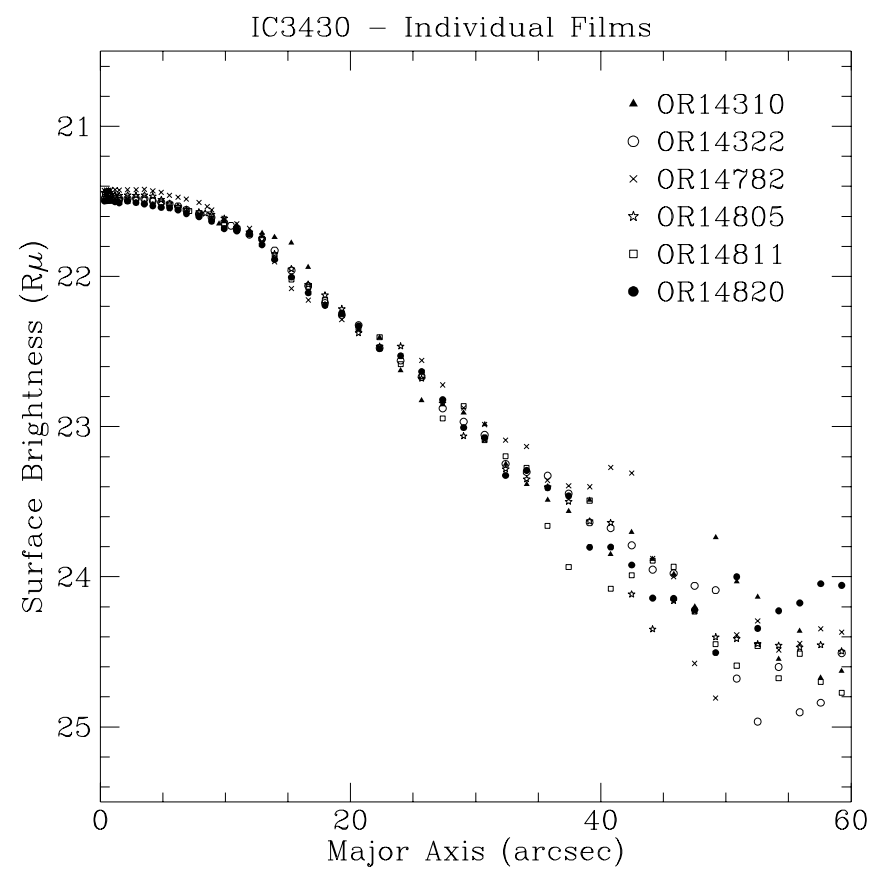

Fig. 1. Radial profiles of IC3430, obtained from the six individual films

apart on the $5^{\circ} \times 5^{\circ}$ area also gives us confidence that the scaling factors derived for each film can later be used for any of the full survey data, irrespective of its location.

Table 2. Raw-frame statistics and scaling factors used. The second and third columns show the sky background counts and $1-\sigma$ noise, the fourth gives the number of counts in the galaxy's profile at 20 arcsec, and the last column shows the multipicative scaling factors used

\begin{tabular}{|c|cc|c|c|}
\hline \multirow{2}{*}{ Film } & \multicolumn{2}{|c|}{ Raw Frame } & \multicolumn{2}{c|}{ IC3430 } \\
\cline { 2 - 5 } Number & Sky & Noise & Profile & Scaling \\
\hline 14310 & 10349 & 520.7 & 6362 & 1.000 \\
14322 & 4553 & 260.3 & 2014 & 3.096 \\
14782 & 1329 & 92.1 & 528 & 12.038 \\
14805 & 2605 & 178.5 & 1162 & 5.634 \\
14811 & 2761 & 186.2 & 1231 & 5.155 \\
14820 & 820 & 59.8 & 354 & 17.783 \\
\hline
\end{tabular}

Now that all frames have been scaled multiplicatively, i.e. each calibrating galaxy has a profile corresponding to the same number of counts (above sky) in each film, it is necessary to scale them additively so that all the background levels also correspond to the same number of counts. We have arbitrarily chosen the background to have a value of 5000 counts - this has no effect on any other calibration procedure. 


\subsection{Alignment of frames}

The last step before median-stacking our set of images is to align them. Shifting of the frames was done using CCDALIGN, which is part of the CCDPACK reduction package (Draper 1993). It is an interactive program which allows all kinds of coordinate transformations, i.e. shifts, stretches and rotations. A reference frame is chosen and a number of objects (usually 5-10) selected by eye. Faint, unsaturated stars are chosen wherever possible to allow an easy determination of their centroid. The program automatically locates the corresponding objects in the other 5 frames and performs a full 5-parameter fit to calculate the new coordinates of each frame. The information is then passed on to another routine which actually transforms the image coordinates. Pairs of registered frames are then co-added to verify the proper running of the program. Typical stretching and rotation factors were of the order of $10^{-4}$ or smaller.

\subsection{Median stacking}

Finally, the six transposed, fully reduced frames were stacked together by means of the MAKEMOS program from CCDPACK. This simply creates a frame where each pixel value is the (unweighted) median of the values in the six separate films. We initally experimented with both averaging and median-stacking, finding no significant difference in the depth reached using the two methods. Medianstacking was therefore favoured over averaging, as it offers the considerable advantage of removing most artifacts present on single films (e.g. satellite trails, scratches or dust adhering to the films during the scanning process). This turns out to be particularly important if we wish to avoid the automated detection of large numbers of spurious faint objects (see Sect. 3.4). Of course strongly variable objects or potentially interesting transient phenomena are also removed by this process - but these are of no interest in the context of the present study. Median-stacking has been extremely effective in removing such artifacts, with all the non-variable, non-stationary or non-astronomical features disappearing completely in the process. We illustrate this in Fig. 2, where a region around VCC1017 has a satellite trail and several scratches on one film (Fig. 2a), none of which can be seen on the median-stack (Fig. 2b).

\subsection{Calibration of median-stacked frames}

Our surface brightness zero-point has been determined in the same way as for the single films (Sect. 2.3), by matching the profile of IC3430 on the median-stacked frame to the CCD-calibrated profile in PP93. The same zero-point can then be used for all other frames, since the scaling factors are the same everywhere.

Comparing the depth reached by single films to that reached by the median stack, we find a gain of approximately 1 magnitude, in agreement with the expected the- oretical gain of $2.5 \log \sqrt{6}$ if all films were equally good (cf. Bland-Hawthorn et al. 1993). The $1-\sigma$ sky noise is at $25.0 \mathrm{R} \mu$ on average for single films and $26.0 \mathrm{R} \mu$ for the median-stack. These values are not quite as deep as suggested by the analysis in SPP95, due to differences in the way the films were scanned by COSMOS and now by SuperCOSMOS: with the original COSMOS data (PP93 and SPP95), the lower scanning resolution effectively smoothed the data on scales of order $1^{\prime \prime}$, resulting in a significant gain in depth. The noise levels would actually be in agreement if the new data were smoothed to $18 \mu \mathrm{m}$ (compared to the nominal original scanning spot of $16 \mu \mathrm{m}$, the slight difference being due to the low-level "halo" of the old spot). Although this natural smoothing does not occur in our present SuperCOSMOS scans, the greater depth can be regained by applying a smoothing function to the co-added frames, as described in Sect. 3.1.

\section{Properties of the median-stacked images}

With our five frames fully reduced and calibrated, we can now proceed to examine several of their properties before and after median-stacking. In particular we examine changes in the background noise and in the point spread function, as well as the change in the numbers of detected objects relative to single films.

The results of median stacking in terms of galaxy profiles can be seen in Fig. 3, which show profiles of VCC 952, a small, moderately low surface brightness galaxy from the catalogue of Binggeli et al. (1988). This galaxy has an extrapolated central surface brightness $21.4 \mathrm{R} \mu$ and exponential scale-length $5.0^{\prime \prime}$. Profiles are shown both from the median-stack (solid line) and from individual films, indicated by different symbols. We have chosen to profile a smaller galaxy rather than one of the five previously mentioned, so as to avoid the degrading effect of medianfiltering on the outer parts of these relatively large galaxies (Sect. 2.2). It can be seen from Fig. 3 that the medianstacked profile is generally a good representation of all the individual profiles, although at very faint levels the uncertainties in the determination of the sky background are such that the individual profiles are equally likely to drop to zero or flatten out. Furthermore, in the range $15^{\prime \prime}$ to $30^{\prime \prime}$ the median-stack profile follows the faintest of the single profile points. This occurs because profile intensities are determined as mean values around an ellipse, rather than median values. They are therefore more susceptible to the inaccuracies present in the single films (witness the large point-to-point scatter present in each of the single profiles), while the profile from the median-stacked frame provides an overall better representation of the true radial profile of a galaxy. 

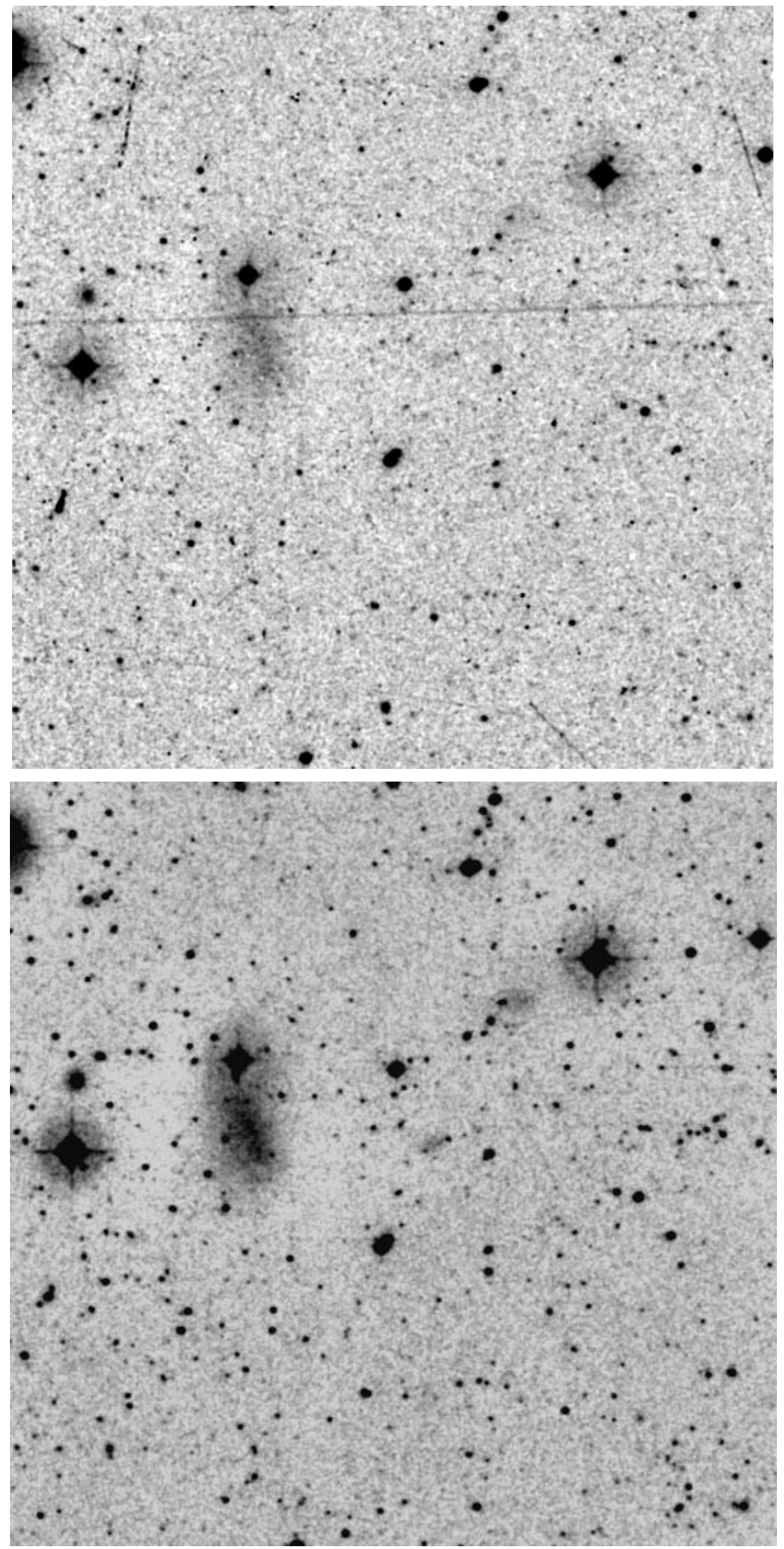

Fig. 2. An $11^{\prime} \times 11^{\prime}$ region around VCC 1017, from a) single film, b) median stack. North is at the top, east to the left. Note the effectiveness of median stacking in removing spurious objects such as satellite trails and scratches, as well as in confirming the reality of faint, low surface brightness galaxies. The two small galaxies west and northwest of VCC 1017 are VCC 993 and VCC 983 respectively, from the catalogue of Binggeli et al. (1985) 


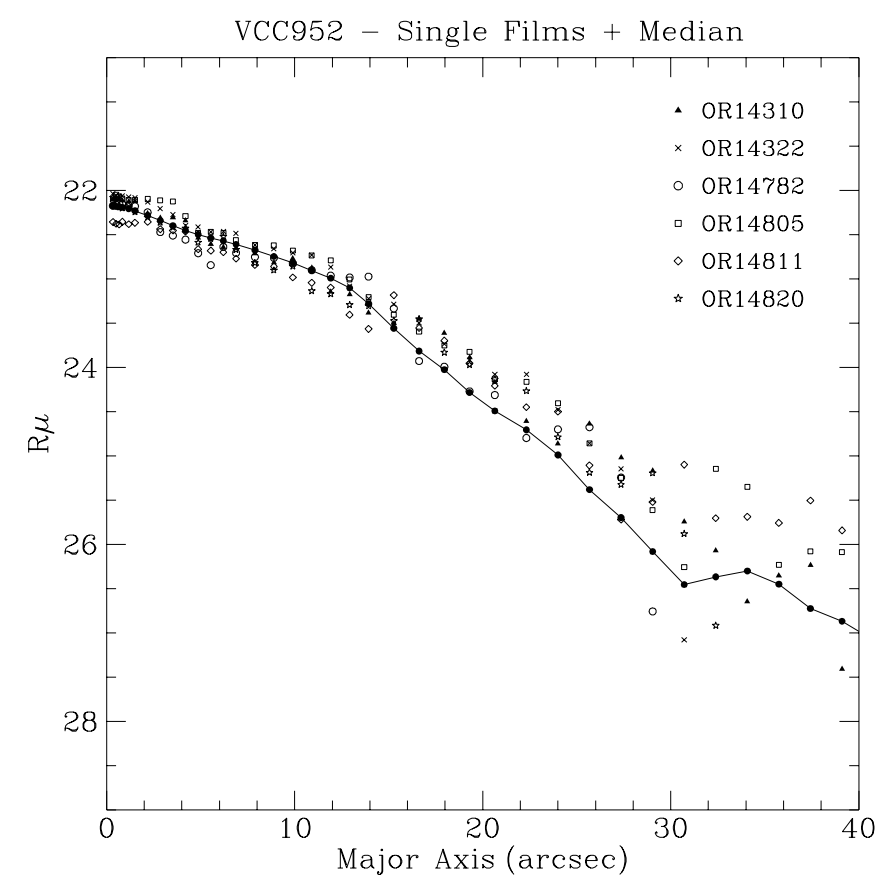

Fig. 3. Profile of VCC 952, showing a comparison between individual film profiles and median-stack profile (solid line)

\subsection{Background noise and smoothing}

Figure 4 shows histograms of the pixel values of an uncrowded region of sky (approximately $3^{\prime} \times 3^{\prime}$ ), from a single film (a) and from the median (b). The noise in the sky is reduced from 426 counts to 180 , in keeping with the expectations from poissonian statistics. We do not find evidence for statistical distortion in our data, which would render the pixel-to-pixel noise non-poissonian. The finer grain size of Tech Pan could explain the absence of distortion, which has been observed on III-a data for scanning apertures smaller than $12 \mu \mathrm{m}$ (Bland-Hawthorn et al. 1993). We might note also that calibration and flatfielding procedures can lead to non-gaussian or even sparse histograms if multiplicative factors stretch or clump the grey levels - although this has no adverse effect on any subsequent use of the data.

The unweighted median approach which we have adopted naturally accounts for the different noise levels on the various films, since poorer films are more likely to have pixel values away from the mean sky level and thus are less likely to contribute to the median value of the six. This was found to be the case when we experimented with adding in two noisier films (other than the six listed in Table 1). This caused virtually no change to the final noise level. Note, though, that in a case where one film was of very low noise compared to the others it would be possible to increase the noise by stacking rather than reduce it: a majority of the films could all, by chance, have a high or low value in a given pixel, so that the "correct" value from the best film would not be chosen as the median. This risk was averted by only including our six best films, which all had similar noise levels to within a factor $\sim 1.4$.

In order to further reduce the pixel-to-pixel noise in our frames, we have also applied a gaussian smoothing function of width 3 pixels $\left(2^{\prime \prime}\right)$ at half-maximum to each median-stacked frame. This had the effect of reducing the noise by a factor $\sim 2$, while having no restrictive effect on object detection (our objects of interest will have minimum exponential scale lengths of $3^{\prime \prime}$, see SPP95).

\subsection{Stellar profiles}

It is worth asking whether the registering and medianstacking processes degrade the resolution of our final image. Although we do not expect registration of the frames to contribute significantly to this effect, different seeing conditions on each exposure could affect the final product. Once again we benefit from the advantages of medianstacking over co-adding, as it reduces the effect of any one film's poorer seeing on the final image (since the pixel values of a compact image will be systematically lower in that film, and will therefore have a lesser effect on the median).

Comparing the radial profiles of small, unsaturated stars on all single films and on the medianed frames, we find no significant degradation. The 'seeing' values quoted in Table 1 are the average FWHM, in arcseconds, of gaussian profiles fitted to a number $(>30)$ of stars in each frame. The seeing measured in the same way on medianstacked frames was $2.7^{\prime \prime}$, corresponding exactly to the median value of the seeings listed in Table 1.

\subsection{Automated galaxy detection}

We now examine the effect of median stacking on the number of objects detected through connected pixel algorithms (Schwartzenberg et al. 1995b). On the one hand we expect the number of real detections to increase on the medianed frame, simply due to the deeper limit reached. On the other hand, however, the number of spurious noise objects present on single films may decrease significantly in the median, depending on the specific signal-to-noise and isophotal limits employed (cf. Driver et al. 1994). For instance if we chose the same limits relative to the noise level, e.g. 9 connected pixels at least $2.5 \sigma$ above sky, we should find similar numbers of noise detections if the noise is genuinely gaussian and uncorrelated from pixel to pixel. This, however, turns out not to be the case. Large numbers of spurious objects are detected on single films. These are due not only to random noise, but also to features such as satellite tracks, scratches or holes in the emulsion and dust particles adhering to the film during scanning (though rigorous attempts are made to clean the films beforehand). These features can be mistaken for small, faint objects when running detection software (although they can be removed at a later stage by applying cuts in the 
Histogram of pixel count value versus frequency.

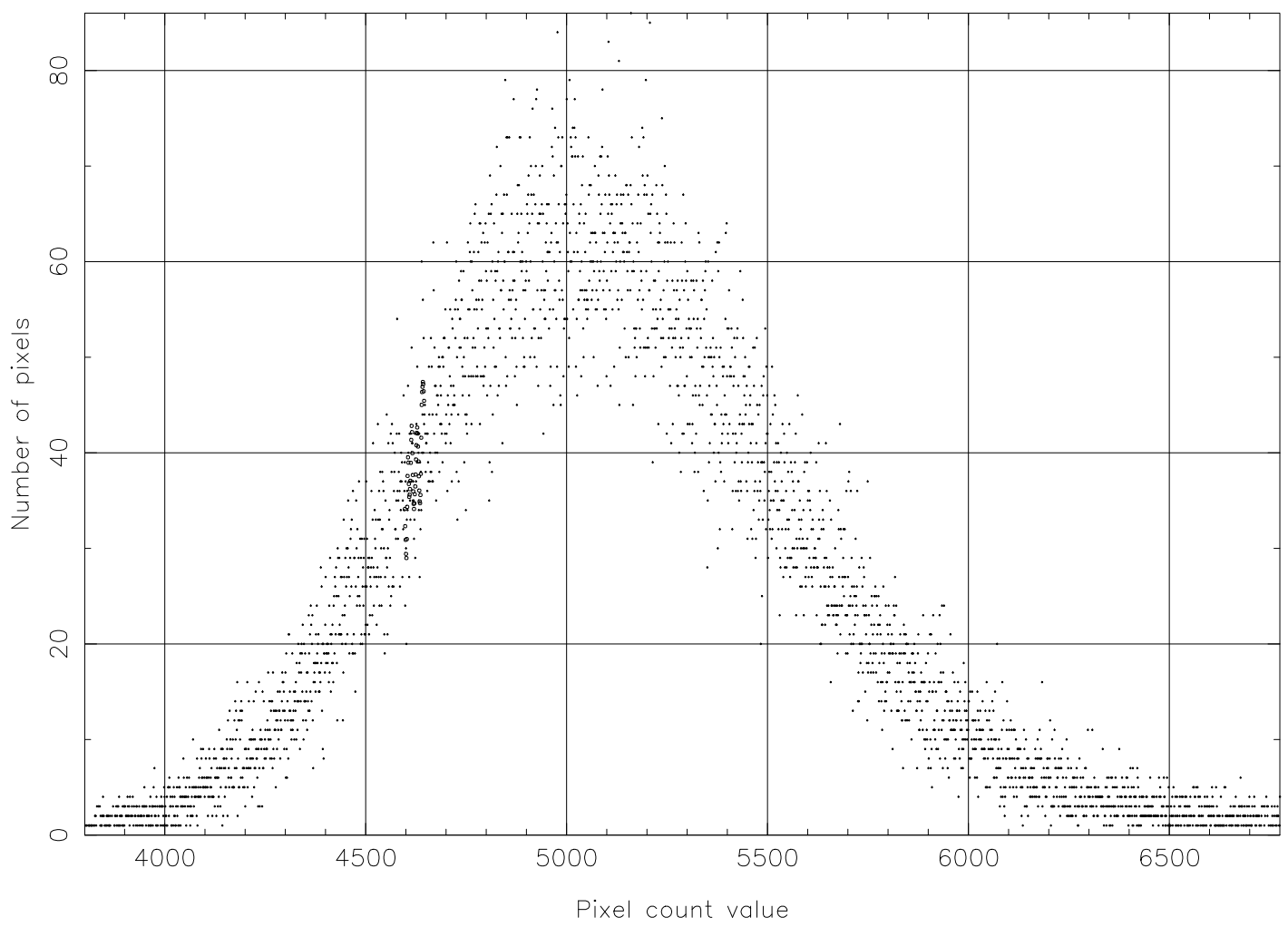

Histogram of pixel count value versus frequency.

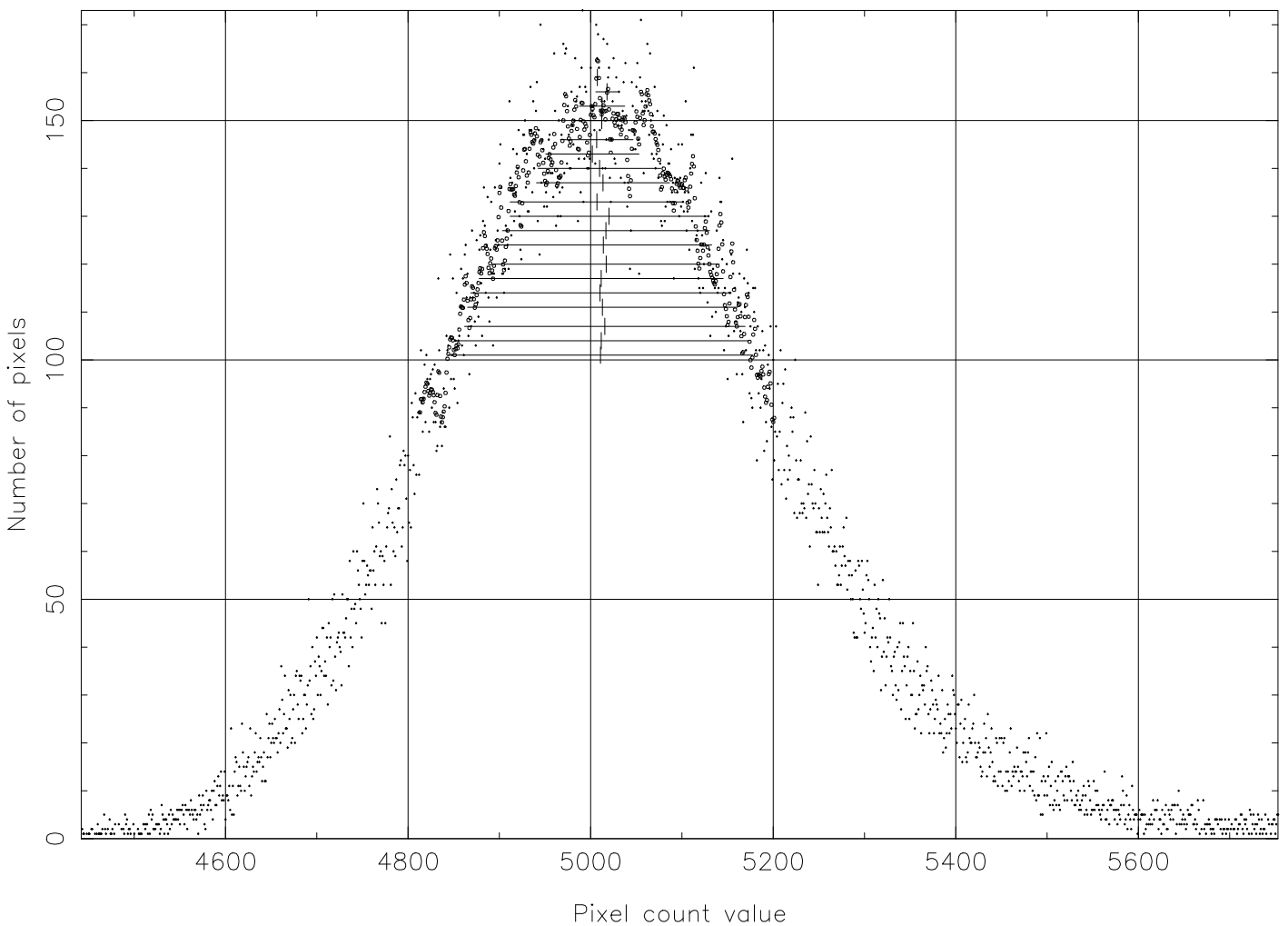

Fig. 4. Sky background histogram of an uncrowded $3^{\prime} \times 3^{\prime}$ region, from a) single film and b) median-stack. Horizontal lines and vertical tick marks near the peaks indicate the width and centre of the distributions at various frequencies 

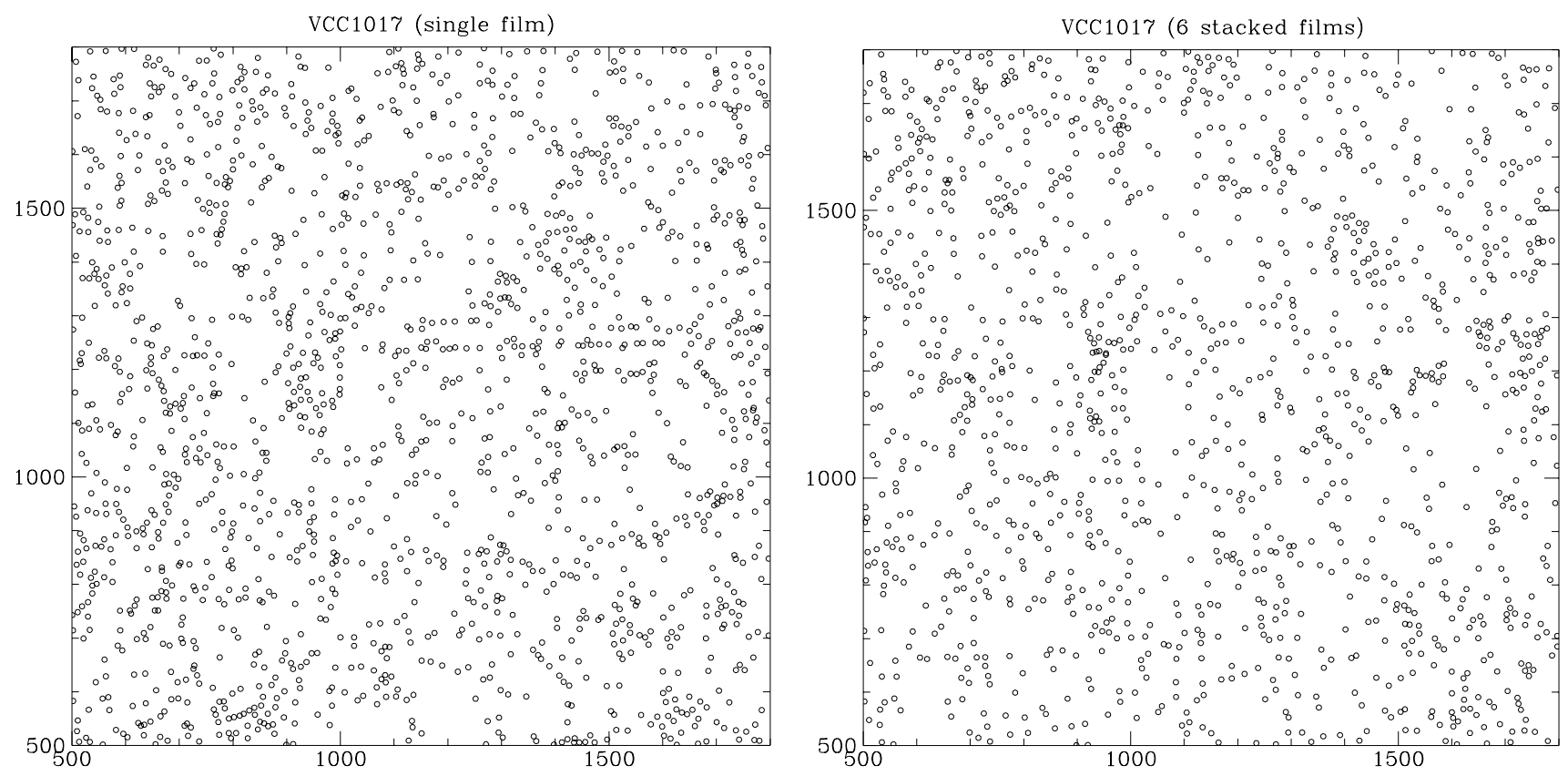

Fig. 5. All objects detected in the region shown in Fig. 2. Note the strings of detections on single film caused by satellite trails a), which do not appear on the median stack b)

magnitude-area plane, as described in Schwartzenberg et al. 1995b). As an example, a satellite trail through one of our films (OR14805) created a "string" of some $50 \mathrm{spu}-$ rious objects. This string is seen across Fig. 5a, where we have plotted all the "objects" detected in the vicinity of VCC1017 (the same region as Fig. 2). Again these spurious objects are not detected in the median stack (Fig. 5b).

The effect of median stacking on detections in terms of the increased depth can also be seen in Fig. 2. Note particularly the two very diffuse, low surface brightness galaxies which appear on the median, to the west and north-west of VCC1017. They can be seen on the single film, but are much more clearly defined in the median-stacked image. These two galaxies, VCC983 and VCC993, were among the faintest to be included in the Virgo Cluster Catalogue (Binggeli et al. 1985), compiled from visual inspection of larger scale photographic plates taken on a $2.5 \mathrm{~m}$ telescope.

\section{Summary}

We have presented the techniques and first results of coadding digitised scans of Tech Pan films for the purpose of detection and photometry of faint and low surface brightness galaxies. After carrying out the necessary reduction and calibration procedures (paying particular attention to sky subtraction and to the scaling of all films to a common level), we have experimented with both median stacking and ordinary averaging. While the same photometric limits are reached in both cases, median stacking is considerably more effective in removing spurious features present in single films. The gain in depth obtained from stacking six films is found to be equivalent to the expected poissonian gain of $\approx 1$ magnitude.

Comparison of the photometric properties of our data before and after stacking show that the background noise is reduced by the amounts expected from poissonian statistics, while an examination of stellar profiles shows no degradation in image size due to the stacking process.

Surface brightness profiles of small, faint galaxies obtained from the median stacked frames appear to be a fair representation of all single film profiles (though of course extending to fainter photometric limits), while the reduced sky noise also allows the profiles to be traced to considerably larger radii. Additionally, the elimination through median stacking of large numbers of spurious objects enables a more efficient use of automated galaxy detection algorithms, making possible the detection of vast numbers of new, uncatalogued low surface brightness galaxies in the Virgo cluster. The generation of such a new catalogue, and its impact on our understanding of low surface brightness galaxies and the Virgo cluster luminosity function, will be the subject of future papers.

Acknowledgements. We are grateful to the UK Schmidt Telescope for supplying the films for this project, and we thank Lance Miller and Harvey MacGillivray for helping obtain scans with the SuperCOSMOS machine at ROE. 


\section{References}

Binggeli B., Sandage A., Tammann G.A., 1985, AJ 90, 1681

Bland-Hawthorn J., Shopbell P.L., Malin D.F., 1993, AJ 106, 2154

Cawson M.G.M., Kibblewhite E.J., Disney M.J., Phillipps S., 1987, MNRAS 224, 557

Currie M., 1992, Starlink User Note, No. 95.8

Davies J.I., Disney M.J., Phillipps S., Boyle B.J., Couch W.J., 1994, MNRAS 269, 349

Draper P.W., 1993, Starlink User Note, No. 139.3

Driver S.P. Phillipps S., Davies J.I., Morgan I., Disney M.J., 1994, MNRAS 266, 155

Hawkins M., 1991, in IAU Wide Field Imag. Newslett. 1, 23

Impey C.D., Bothun G.D, Malin D.F., 1988, ApJ 330, 634

Irwin M.J., Davies J.I., Disney M.J., Phillipps S., 1990, MNRAS 245, 289
Jacoby G.H., et al., 1992, PASP 104, 599

Kemp S.N., Meaburn J., 1993, A\&A 274, 19

MacGillivray H.T., Stobie R.S., 1984, Vistas Astron. 27, 433

Miller L.A., Cormack W., Paterson M., Beard S., Lawrence L., 1992, in Digitised Optical Sky Surveys. In: MacGillivray H.T., Thomson E.B. (eds.). Kluwer, Dordrecht, p. 133

Parker Q.A. , Phillipps S., Morgan D.H., Malin D.F., Russell K.F., Hartley M., Savage A., 1994, in Astronomy from Wide-Field Imaging. In: MacGillivray et al. (eds.). Kluwer, Dordrecht, p. 129

Phillipps S., Parker Q.A., 1993, MNRAS 265, 385 (PP93)

Schwartzenberg J.M., Phillipps S., Parker Q.A., 1995a, A\&A 293, 332 (SPP95)

Schwartzenberg J.M., Phillipps S., Smith R.M., Couch W.J., Boyle B.J., 1995b, MNRAS 275, 121 